\title{
Surgical Outcomes of Nontraumatic Pediatric Cataracts
}

\author{
Aslihan Uzun, Huban Atilla \\ Department of Ophthalmology, Training and Research Hospital, Ordu University, Ordu, Turkey \\ Email: draslihanuzun@gmail.com
}

How to cite this paper: Uzun, A. and Atilla, H. (2020) Surgical Outcomes of Nontraumatic Pediatric Cataracts. Open Journal of Ophthalmology, 10, 115-122. https://doi.org/10.4236/ojoph.2020.102013

Received: April 4, 2020

Accepted: May 5, 2020

Published: May 8, 2020

Copyright $\odot 2020$ by author(s) and Scientific Research Publishing Inc. This work is licensed under the Creative Commons Attribution International License (CC BY 4.0).

http://creativecommons.org/licenses/by/4.0/

\section{(c) (i) Open Access}

\begin{abstract}
Purpose: To evaluate post-surgical complications in patients who were operated for nontraumatic pediatric cataracts in our clinic. Methods: Medical records of 62 patients (101 eyes) that underwent cataract surgery younger than 15 years of age, without history of ocular trauma and with a minimum follow-up period of 6 months, were reviewed retrospectively. Results: The most common initial complaint was leukocoria. Thirty-nine patients $(62.9 \%)$ had bilateral cataracts and 23 patients $(37.1 \%)$ had unilateral cataracts. The most common type was posterior polar cataract $(22 \%)$ in patients with unilateral cataracts, and total cataract (33\%) in patients with bilateral cataracts. The overall prevalence of postoperative complications was $58 \%$, visual axis opacification (VAO) being the most common one (39\%). The incidence of VAO was significantly higher in eyes with intact posterior capsules. Secondary glaucoma occurred in $12(12 \%)$ eyes. Thirty-one (50\%) patients were orthophoria, $17(27 \%)$ patients had esotropia, and 14 (23\%) patients had exotropia. Additional surgery for all of these complications was performed in 53 (53\%) eyes. Conclusions: Despite appropriate surgical treatment of nontraumatic pediatric cataracts, post-surgical complications including VAO, glaucoma, or strabismus remain an important cause of morbidity in these patients. Posterior capsulotomy and anterior vitrectomy must be performed in all children below 6 years to reduce the need for additional surgery for VAO.
\end{abstract}

\section{Keywords}

Congenital Cataract, Pediatric Cataract, Visual Axis Opacification, Glaucoma, Surgery

\section{Introduction}

Pediatric cataracts are one of the main causes of treatable visual loss in childhood worldwide [1]. Congenital cataracts accounting for $5 \%$ to $20 \%$ of pediatric 
blindness, are the main type of pediatric cataracts [1] [2]. The prevalence of congenital cataract has been reported as 3.0 - 4.5 per 10,000 live births in the first year of their life [3]. Because early childhood is the critical period of visual development, early diagnosis and appropriate removal of cataract are required in cases with congenital cataracts [4] [5]. Visual axis must be cleared before 6 weeks of age for unilateral cataracts and not later than 10 weeks in bilateral cataracts [4].

Management of pediatric cataracts is challenging, important and quite different from adult cataracts. Compared to adult patients, anterior lens capsule is much more elastic, cataract is much softer, posterior capsule opacification rate is higher, and anterior vitreous can serve as a scaffold for proliferation of lens epithelial cells in children [6]. Pediatric eyes have higher inflammatory activity that can lead to posterior synechiae, seclusio pupilla, iris bombe and secondary angle closure glaucoma. Duration of visual deprivation (timing of surgery), aphakia correction (spectacles, contact lenses), intraocular lens (IOL) implantation, biometric calculation, axial growth of pediatric eye resulting in refractive shift and amblyopia treatment are also controversial issues in the management of pediatric cataracts [4].

Pediatric patients are more susceptible to develop complications that can lead to deprivation and visual loss following cataract surgery compared to adult patients, consequently. In the current study, the aim was to evaluate post-surgical complications in patients who were operated for nontraumatic pediatric cataracts in our clinic. Moreover, this study aimed to examine the interrelationships of outcomes with other variables such as patient age.

\section{Materials and Methods}

Medical records of 62 pediatric patients who underwent cataract surgery at Ankara University, Faculty of Medicine, Department of Ophthalmology, Pediatric Ophthalmology and Strabismus Section were reviewed retrospectively. The study protocol was approved by the Research Ethics Committee of Ankara University Faculty of Medicine (approval no. 15-514-12). The study procedures were performed in accordance with the ethical standards as laid down in the 1964 Declaration of Helsinki and its later amendments or comparable ethical standards.

The inclusion criteria were as follows: 1) being under the age 15,2 ) having surgery for unilateral or bilateral cataracts, 3) not to have a history of ocular trauma, 4) undergoing surgery at our clinic and 5) follow up period of at least 6 months after surgery. All children underwent complete ocular evaluation and wherever necessary, examination under sedation was performed. Cataract and complication surgeries were performed by two surgeons. The demographics and clinical characteristics of the patients, the complaints of parents, family history, consanguinity, surgical technique, optical rehabilitation, post-surgical complications and management of these complications were recorded. Complications were considered early if they occurred within 3 months after surgery and delayed if they occurred 3 months after surgery. 
Postoperative optical rehabilitation involved the use of spectacles or contact lenses. The power of the spectacles was determined by careful retinoscopy.

\section{Results}

One-hundred one eyes of 62 patients [34 (55\%) males and 28 (45\%) females] were included in this retrospective study. Thirty-nine (63\%) patients had bilateral cataracts and 23 (37\%) had unilateral cataracts. The mean age at the time of diagnosis was $10.23 \pm 16.09$ months (range, 1 - 92 months) in patients with bilateral cataracts and $31.65 \pm 31.36$ months (range, 1 - 96 months) with unilateral cataracts. The mean age at the time of surgery was $13.15 \pm 20.60$ months (range, $1-101$ months) in patients with bilateral cataracts and $33.26 \pm 31.36$ months (range, 1 96 months) with unilateral cataracts. The mean follow-up period was 32 months.

Seventeen $(28 \%)$ patients had a positive family history of pediatric cataracts and $8(13 \%)$ patients had a positive history of parental consanguinity. Fifty-five ( $88 \%)$ patients presented with some complaints, one patient (2\%) was diagnosed prenatally, and 6 patients (10\%) were diagnosed during routine examination without any complaints. Main complaints of the parents were leukocoria (37\%), strabismus (24\%), nystagmus (12\%), lack of fixation (12\%), visual loss (12\%), and microphthalmia $(3 \%)$. While $31(80 \%)$ of 39 patients with bilateral cataracts had nystagmus, only 2 (9\%) of 23 patients with unilateral cataracts had nystagmus. The comparison between bilateral and unilateral cases was given in Table 1.

The most common types of cataracts were total (28\%), nuclear (19\%) and posterior polar (14\%) cataract in all patients (Table 2). The most frequent morphology was posterior polar cataract $(22 \%)$ in patients with unilateral cataracts, and total cataract $(33 \%)$ in patients with bilateral cataracts. While none of the patients with bilateral cataracts had persistent fetal vasculature (PFV), 22\% of patients with unilateral cataracts had PFV.

During surgery, anterior capsulotomy was performed with Continuous Curvilinear Capsulorhexis (CCC) in all eyes. Lens aspiration/extraction was performed with irrigation/aspiration in 75 (74\%) eyes, and with phacoemulsification in 26 (26\%) eyes. Cataract surgery was combined with posterior capsulotomy and anterior vitrectomy in 84 (83\%) eyes of patients under 4 years of age. Twenty-four (24\%) eyes had primary IOL implantation and secondary IOL was implanted in 22 eyes $(22 \%)$. Thirty-eight (61\%) of all patients underwent cataract surgery in the first year of their life. In these patients, cataract surgery was routinely combined with anterior vitrectomy and posterior capsulotomy. None of these patients had primary IOL implantation combined with cataract surgery. On the other hand, all eyes of patients older than 4 years underwent primary IOL implantation and $59 \%$ of eyes of patients between 13 and 48 months of age had primary IOL implantation.

The overall incidence of postoperative complications including visual axis opacification (VAO, 39\%), secondary glaucoma (12\%), pupillary membrane (5\%) and anterior segment hemorrhage (2\%) was 58\%. Moreover, $64 \%$ of all post-surgical complications occurred in eyes of patients who underwent surgery in the first 
year of their life. The distribution of post-surgical complications by age was given in Table 3. Additional surgery for these complications was performed in 53 (53\%) eyes. The most common types of cataracts in the eyes that developed postoperative complications were total and nuclear (24\% and 15\%, respectively).

Table 1. Comparison between unilateral and bilateral cases.

\begin{tabular}{ccc}
\hline & Bilateral cases & Unilateral cases \\
\hline No. of cases & $39(63 \%)$ & $23(37 \%)$ \\
mean age of diagnosis (months) & $10.23 \pm 16.09$ & $31.65 \pm 31.36$ \\
mean age of surgery (months) & $13.15 \pm 20.60$ & $33.26 \pm 31.36$ \\
parental consanguinity & $8(20 \%)$ & 0 \\
Family history of cataract & $14(36 \%)$ & $3(13 \%)$ \\
Nystagmus & & $2(9 \%)$ \\
Preoperative & $27(69 \%)$ & $2(9 \%)$ \\
Postoperative & $31(80 \%)$ & \\
Strabismus & & $8(35 \%)$ \\
Preoperative & $14(36 \%)$ & $11(48 \%)$ \\
Postoperative & $20(51 \%)$ & \\
\hline
\end{tabular}

Table 2. Morphological types of cataracts.

\begin{tabular}{cc}
\hline Cataract Morphology & No. of eyes \\
\hline Total Cataract & $29(28 \%)$ \\
Nuclear Cataract & $19(19 \%)$ \\
Posterior Polar Cataract & $14(14 \%)$ \\
Posterior Subcapsular Cataract & $8(8 \%)$ \\
Nuclear \& Anterior Polar Cataract & $7(7 \%)$ \\
Cortical Cataract & $5(5 \%)$ \\
PFV + Posterior Polar Cataract & $5(5 \%)$ \\
Anterior Polar Cataract & $4(4 \%)$ \\
Nuclear \& Posterior Polar Cataract & $3(3 \%)$ \\
Membranous Cataract & $2(2 \%)$ \\
Punctate Cataract & $2(2 \%)$ \\
Lamellar Cataract & $2(2 \%)$ \\
Sutural Cataract & $1(1 \%)$ \\
Total & $101(100 \%)$
\end{tabular}

PFV: Persistent Fetal Vasculature.

Table 3. The distribution of post-surgical complications by age (month).

\begin{tabular}{ccccc}
\hline & $\mathbf{1}-\mathbf{1 2} \mathbf{M}$ & $\mathbf{1 3 - 4 8} \mathbf{M}$ & $\mathbf{4 9}-\mathbf{1 0 8} \mathbf{M}$ & Total \\
\hline No. of eyes & 68 & 22 & 11 & 101 \\
Post-surgical complications & 37 & 11 & 10 & 58 \\
Anterior segment hemorrhage & 2 & 0 & 0 & 2 \\
Pupillary membrane & 2 & 2 & 1 & 5 \\
Visual axis opacification & 22 & 8 & 9 & 39 \\
Secondary glaucoma & 11 & 1 & 0 & 12
\end{tabular}


In the early postoperative period, one of the 5 eyes with pupillary membrane received peribulbar steroid injection, 1 patient was given oral steroid treatment, and in the remaining 3 eyes, frequency of topical steroids was increased. Pupillary membrane resolved in all patients. Anterior segment hemorrhages that were diagnosed at early postoperative period in 2 eyes, resolved spontaneously.

In all eyes, VAO developed in $26(31 \%)$ of 84 eyes that received posterior capsulotomy and anterior vitrectomy, and in 13 (77\%) of 17 eyes with an intact posterior capsule. The incidence of VAO was higher in eyes that did not receive posterior capsulotomy and anterior vitrectomy. Additional surgeries including synechotomy, anterior vitrectomy and posterior capsulotomy were performed in 21 eyes, and Neodymium: YAG laser capsulotomy was applied in 10 eyes for the treatment of VAO. The remaining 8 eyes with VAO did not require additional treatment as retinoscopy could be performed in spite of the opacification.

Although posterior capsulotomy and anterior vitrectomy was performed in $94 \%$ of eyes of $1-12$ months group, VAO rate in this group was $32 \%$. None of patients older than 49 months had posterior capsulotomy and anterior vitrectomy, $82 \%$ of these eyes developed VAO, consequently. While additional surgery for VAO was performed in $77 \%$ of eyes in $1-12$ months group, all patients undergoing Neodymium: YAG laser capsulotomy were older than 72 months of age.

Secondary glaucoma developed in 12 (12\%) aphakic eyes, postoperatively. In 3 (25\%) of 12 eyes, peripheral iridectomy was performed as a secondary intervention. Nine eyes (75\%) had primary open-angle glaucoma. Trabeculectomy was performed in $2(17 \%)$ eyes and antiglaucomatous medications were prescribed in 7 (58\%) eyes with secondary glaucoma. Additionally, $92 \%$ of eyes with secondary glaucoma was the patients who underwent cataract surgery in the first year of their life. None of the patients who had surgery after 49 months developed glaucoma.

The residual refractive errors of $46(46 \%)$ patients who had primary or secondary IOL implantation, were corrected with spectacles. Refractive errors of 48 aphakic patients first corrected with spectacles. Although contact lenses were prescribed in 7 aphakic patients who had surgery for unilateral cataracts, contact lenses had to be changed to spectacles, because of noncompliance. Furthermore, occlusion therapy was given to $26(26 \%)$ patients with unilateral cataracts, anisometropia or strabismus.

In the postoperative period, 31 (50\%) of all patients were orthophoria, 17 (27\%) patients had esotropia, and 14 (23\%) patients had exotropia. Surgical treatment was performed in 6 patients for esotropia and in 2 patients for exotropia. When we evaluated the distribution of postoperative strabismus by age, we found that $78 \%$ of all patients with strabismus were the ones who had surgery before 12 months of age. Moreover, $63 \%$ of patients who underwent cataract surgery in the first year of life, developed strabismus.

\section{Discussion}

Early diagnosis and prompt referral to surgery are crucial to prevent deprivation amblyopia and to achieve a good visual outcome in patients with pediatric cata- 
racts [7] [8]. Pediatric eyes have mitotically active lens epithelial cells leading to higher incidence of VAO [9]. The inflammatory response is severe in children and fibrous membranes that form on the intact anterior vitreous acting as a scaffold result in VAO. Opacified posterior capsule is also very common after cataract extraction in children, and can be an amblyogenic hazard. Hosal et al. reported that $79 \%$ of eyes with an intact posterior capsule, $43 \%$ of eyes having posterior capsulectomy, $23 \%$ of eyes having posterior capsulectomy and anterior vitrectomy developed VAO following pediatric cataract surgery [9]. The incidence of VAO was reported lower than $10 \%$ in patients who had congenital cataract surgery combined with posterior capsulotomy and anterior vitrectomy in previous studies [10] [11]. Correspondingly, $82 \%$ of eyes of patients with intact posterior capsules developed VAO, in our study. Formerly, we have not performed posterior capsulotomy and anterior vitrectomy in children that were older than 4 years, routinely. That can be the underlying cause of higher rate of postoperative VAO (39\%). In such cases, strategies to provide a clear visual axis are necessary to achieve visual rehabilitation. Nevertheless pediatric patients can develop $\mathrm{VAO}$ even if a posterior capsulotomy and anterior vitrectomy are performed, anyway [6]. In our study, $32 \%$ of eyes of patients who underwent cataract surgery combined with posterior capsulotomy and anterior vitrectomy in the first year life, developed VAO.

Although the clearance of visual axis, correction of refractive errors and treatment of amblyopia are the keys to a good visual outcome in pediatric cataracts, the avoidance and management of complications are also vital [4]. Kuhli-Hattenbach et al. reported that the incidence of postoperative complications in aphakic patients following congenital cataract surgery was 35\% and $26 \%$ of eyes required additional surgery for complications [10]. In our study, the rate of postoperative complications was $58 \%$ and $53 \%$ eyes required additional surgery for these complications. Furthermore, as seen in Table 2, post-surgical complications were higher in patients who underwent cataract surgery in the first year of their life.

Glaucoma following pediatric cataract surgery can develop in the early postoperative period or as late as decades after surgery [4]. The incidence of glaucoma following congenital cataract surgery was reported as $1 \%-41 \%$ in previous studies [12]-[17]. Different studies have suggested that performing early surgery is a risk factor for the development of glaucoma [10] [12]. Vishwanath et al. reported that the incidence of aphakic glaucoma was significantly higher in patients who had surgery before 4 weeks of life [17]. In our current study, 12 (12\%) of our cases developed glaucoma and they were aphakic. None of the patients who had surgery after 49 months developed glaucoma, and 11 (92\%) of eyes with secondary glaucoma were the eyes of patients who underwent surgery during the first year of life. Lower incidence of glaucoma in our study can be due to our relatively short term follow-up period (32 months).

Park et al. reported that 55\% of patients developed strabismus following bilateral pediatric cataract surgery and age at the time of surgery was significantly 
lower in these patients [18]. Postoperative strabismus rate was 66\% (78\% esotropia, 7\% intermittent esotropia, 7\% exotropia, 7\% intermittent exotropia) in children treated for dense congenital or developmental cataracts and early onset was found to be a significant risk factor for strabismus in Birch et al.'s study [19]. In our study, $50 \%$ of patients were orthophoria, $27 \%$ had esotropia and $23 \%$ had exotropia, postoperatively. Similarly, $78 \%$ of all patients with strabismus were the ones who had surgery in the first year of life, in our study.

The presence of preoperative or postoperative nystagmus has been strongly correlated with worse visual outcome. Delayed surgery results in nystagmus especially in children with bilateral congenital cataracts due to severe visual deprivation [7]. In Magli et al.'s study, nystagmus was present in $26.6 \%$ of patients with bilateral cataracts [20]. In our study, 53\% of patients had postoperative nystagmus. Correspondingly, nystagmus rate was higher $(80 \%)$ in patients with bilateral cataracts. In addition, the mean age at the time of diagnosis was earlier in patients with bilateral cataracts.

Retrospective design and relatively short term follow up (32 months) were the limitations of our study. Nevertheless, this study highlights to the importance of appropriate management of posterior capsule during pediatric cataract surgery. Posterior capsulotomy and anterior vitrectomy must be performed in all children below 6 years to reduce the need for additional surgery. Patients older than 6 years can cooperate for Neodymium: YAG laser capsulotomy. Further studies with longer follow-up period can clarify the exact incidence of post-surgical complications.

\section{Conflicts of Interest}

The authors declare no conflicts of interest regarding the publication of this paper.

\section{References}

[1] Crockett, C., Camero, K.A., Kong, L. and Yen, K.G. (2017) Visual Outcomes of Patients Presenting with Bilateral Infantile Cataracts and Nystagmus. Canadian Journal of Ophthalmology, 52, 203-206. https://doi.org/10.1016/j.jcjo.2016.08.021

[2] Gogate, P., Gilbert, C. and Zin, A. (2011) Severe Visual Impairment and Blindness in Infants: Causes and Opportunities for Control. Middle East African Journal of Ophthalmology, 18, 109-114. https://doi.org/10.4103/0974-9233.80698

[3] Repka, M.X., Dean, T.W., Lazar, E.L., Yen, K.G., Lenhart, P.D., Freedman, S.F., Hug, D., Rahmani, B., Wang, S.X., Kraker, R.T., Wallace, D.K. and Pediatric Eye Disease Investigator Group (2016) Cataract Surgery in Children from Birth to Less Than 13 Years of Age: Baseline Characteristics of the Cohort. Ophthalmology, 123, 2462-2473. https://doi.org/10.1016/j.ophtha.2016.09.003

[4] Lloyd, I.C., Ashworth, J., Biswas, S. and Abadi, R.V. (2007) Advances in the Management of Congenital and Infantile Cataract. Eye, 21, 1301-1309. https://doi.org/10.1038/sj.eye.6702845

[5] Khokhar, S.K., Pillay, G., Dhull, C., et al. (2017) Pediatric Cataract. Indian Journal of Ophthalmology, 65, 1340-1349. https://doi.org/10.4103/ijo.IJO_1023_17 
[6] Batur, M., Gul, A., Seven, E., Can, E. and Yaşar, T. (2016) Posterior Capsular Opacification in Preschool- and School-Age Patients after Pediatric Cataract Surgery without Posterior Capsulotomy. Turkish Journal of Ophthalmology, 46, 205-208. https://doi.org/10.4274/tjo.24650

[7] Medsinge, A. and Nischal, K.K. (2015) Pediatric Cataract: Challenges and Future Directions. Clinical Ophthalmology, 9, 77-90. https://doi.org/10.2147/OPTH.S59009

[8] Vasavada, A.R. and Nihalani, B.R. (2006) Pediatric Cataract Surgery. Current Opinion in Ophthalmology, 17, 54-61.

[9] Hosal, B.M. and Biglan, A.W. (2002) Risk Factors for Secondary Membrane Formation After Removal of Pediatric Cataract. Journal of Cataract \& Refractive Surgery, 28, 302-309. https://doi.org/10.1016/S0886-3350(01)01028-8

[10] Kuhli-Hattenbach, C., Lüchtenberg, M., Kohnen, T. and Hattenbach, L.O. (2008) Risk Factors for Complications after Congenital Cataract Surgery without Intraocular Lens Implantation in the First 18 Months of Life. American Journal of Ophthalmology, 146, 1-7. https://doi.org/10.1016/j.ajo.2008.02.014

[11] Abdelmoaty, S.M.A. and Behbehani, A.H. (2011) The Outcome of Congenital Cataract Surgery in Kuwait. Saudi Journal of Ophthalmology, 25, 295-299. https://doi.org/10.1016/j.sjopt.2011.01.002

[12] Lambert, S.R., Purohit, A., Superak, H.M., Lynn, M.J. and Beck, A.D. (2013) Long-Term Risk of Glaucoma after Congenital Cataract Surgery. American Journal of Ophthalmology, 156, 355-361. https://doi.org/10.1016/j.ajo.2013.03.013

[13] Freedman, S.F., Lynn, M.J., Beck, A.D., et al. (2015) Glaucoma-Related Adverse Events in the First Five Years after Unilateral Cataract Removal in the Infant Aphakia Treatment Study. JAMA Ophthalmology, 133, 907-914. https://doi.org/10.1001/jamaophthalmol.2015.1329

[14] Allen, R.J., Speedwell, L. and Russell-Eggitt, I. (2010) Long-Term Visual Outcome after Extraction of Unilateral Congenital Cataracts. Eye, 24, 1263-1267. https://doi.org/10.1038/eye.2009.295

[15] Yi, K. and Chen, T.C. (2008) Aphakic Glaucoma after Congenital Cataract Surgery. International Ophthalmology Clinics, 48, 87-94. https://doi.org/10.1097/IIO.0b013e3181692d7a

[16] Tatham, A., Odedra, N., Tayebjee, S., Anwar, S. and Woodruff, G. (2010) The Incidence of Glaucoma Following Pediatric Cataract Surgery: A 20-Year Retrospective Study. Eye, 24, 1366-1375. https://doi.org/10.1038/eye.2010.46

[17] Vishwanath, M., Cheong-Leen, R., Taylor, D., Russell-Eggitt, I. and Rahi, J. (2004) Is Early Surgery for Congenital Cataract a Risk Factor for Glaucoma? British Journal of Ophthalmology, 88, 905-910. https://doi.org/10.1136/bjo.2003.040378

[18] Park, S.H., Na, J.H. and Shin, S.Y. (2010) Strabismus Following Bilateral Cataract Surgery in Childhood. Japanese Journal of Ophthalmology, 54, 272-277. https://doi.org/10.1007/s10384-010-0806-0

[19] Birch, E.E., Wang, J., Felius, J., Stager Jr., D.R. and Hertle, R.W. (2012) Fixation Control and Eye Alignment in Children Treated for Dense Congenital or Developmental Cataracts. Journal of AAPOS, 16, 156-160. https://doi.org/10.1016/j.jaapos.2011.11.007

[20] Magli, A., Forte, R. and Rombetto, L. (2013) Long-Term Outcome of Primary versus Secondary Intraocular Lens Implantation after Simultaneous Removal of Bilateral Congenital Cataract. Graefe's Archive for Clinical and Experimental Ophthalmology, 251, 309-314. https://doi.org/10.1007/s00417-012-1979-7 